\title{
Klaus Kastner
}

\section{Rückblick: NSDAP, Reichsparteitage, Rassengesetze}

In Nürnberg, einer Stadt, die an der Wende des Mittelalters zur Neuzeit gelegentlich auch als ,caput Germaniae" bezeichnet wurde, sind seit mehr als neun Jahrhunderten Kunst und Kultur, Handel und Gewerbe und seit der Mitte des 19. Jahrhunderts auch die Industrie „zu Hause“. In der ersten Hälfte des 20. Jahrhunderts war eben dieses Nürnberg für zwei Jahrzehnte - freilich neben anderen deutschen Städten, wie Berlin, München oder Weimar - auch einer der Schauplätze des Nationalsozialismus und damit ein Ort totalitärer Herrschaft.

Drei Begriffe machen für manche den Namen der Stadt Nürnberg geradezu zum Synonym für den Nationalsozialismus, nämlich: die NSDAP, die Reichsparteitage und die Rassengesetze. Ein vierter topos folgt schließlich in den Jahren nach 1945, nämlich die Nürnberger Prozesse.

\section{Die NSDAP}

Es stellt sich als erstes die Frage, wie es dazu kommen konnte, dass eine Stadt, in der beispielsweise bei den Reichstagswahlen vor dem Ersten Weltkrieg die Sozialdemokratische Partei die meisten Stimmen erhielt und in der während der Weimarer Republik die Sozialdemokraten und Kommunisten fast die Hälfte aller Stimmen auf sich vereinigen konnten, sich zu einem Ort entwickelte, der von den Nationalsozialisten gewissermaßen zum Forum für viele ihrer Aktivitäten auserkoren wurde.

Die Wurzel liegt zum einen - wie in vielen Teilen Deutschlands nach 1920 - in dem verlorenen Ersten Weltkrieg, in der darin begründeten wirtschaftlichen Misere und in der Tatsache, dass man sich mit dem Ergebnis des sogenannten Versailler Friedensvertrages nicht abfinden wollte. Bereits 1923 begannen die Nationalsozialisten zusammen mit anderen deutsch-nationalen Gruppierungen, sich in Nürnberg darzustellen. Man beging hier den „Deutschen Tag“. Hitler und seine NSDAP waren damals noch nicht dominierend. Doch der erste Mann der Nürnberger NSDAP, Julius Streicher, ein seit 1909 in Nürnberg lebender Volksschullehrer, der 1922 eine Ortsgruppe der NSDAP in Nürnberg gegründet hatte, war ein Einpeitscher, der sich Hitlers absolutem Führungsanspruch unterworfen hatte. Streicher vertrat in Nürnberg das nationalsozialistische Gedankengut nicht nur persönlich, sondern brachte es demagogisch auch unter die Bevölkerung. Er gab seit 1923 in Nürnberg den „Stürmer" heraus. Diese ursprünglich als allgemein politisches Lokalblatt konzipierte Wochenzeitung - sie gehörte Streicher persönlich, war also keine offizielle Parteizeitung - entwickelte sich im Laufe der Jahre zu einem über ganz Deutschland verbreiteten antisemitischen Hetzblatt mit einer Wochenauflage von meist 500.000. Das Zitat „Die Juden sind unser Unglück“ (es stammte von dem Historiker Heinrich v. Treitschke) prangte auf der Titelseite einer jeden Ausgabe des "Stürmer“. 22 Jahre lang verbreitete dieses Periodikum übelste Diffamierungsparolen gegen den Weltfeind „Alljuda“. Karikaturen, erfundene Geschichten, beispielsweise über Ritualmorde und Hostienschändungen durch Juden, und Berichte über die „Verschwörung des internationalen Finanzjudentums" schürten den Hass gegen alles Jüdische.

Nicht zuletzt dieses planmäßige Hetzen gegen alles Jüdische und das damit verbundene Verhetzen weiter Volksschichten, nicht nur in Nürnberg, sondern auf dem Weg über die Wochenzeitung „Der Stürmer" im gesamten Deutschen Reich, war dann - ich greife jetzt 20 Jahre weiter - der maßgebliche Grund dafür, dass das Internationale Militärtribunal in Nürnberg im Oktober 1946 Julius Streicher wegen Verbrechen gegen die Menschlichkeit (Straftatbestand vier im Sinne der Anklage) zum Tod durch den Strang verurteilte.

Streicher gründete in Nürnberg am 20.10.1922 die erste Ortsgruppe der NSDAP außerhalb von München. Als treuer Vasall Hitlers "trommelte" er wie kein zweiter für die nationalsozialistische "Bewegung", so dass die NSDAP in Nürnberg im Jahre 1927 schon 2000 eingeschriebene Parteimitglieder hatte. Bei der bayerischen Landtagswahl am 24. April 1932 wurde die NSDAP in Nürnberg mit 37,6 \% der Stimmen erstmals die stärkste politische Kraft. Doch eine demokratisch legitimierte, absolute Mehrheit errang die NSDAP in Nürnberg nie. Bei der Reichstagswahl am 5. März 1933, der letzten freien Wahl, bekam die NSDAP in Nürnberg nur $41,7 \%$ der Stimmen, während es im 
Reichsdurchschnitt immerhin 43,9\% waren. Also: Nürnberg war keineswegs eine Stadt, in welcher der Nationalsozialismus vor 1933 politisch dominierend war.

Als aber der Nationalsozialismus in Berlin die Regierungsgewalt errungen hatte, ging es auch in Nürnberg mit den demokratischen Rechten rasch dahin. Nach den "Gleichschaltungsgesetzen" verhafteten die Nationalsozialisten sofort den Nürnberger Oberbürgermeister Luppe und Bürgermeister Treu. Der zugleich entfachte „braune Terror“ hatte es auf alle Repräsentanten der Demokratie abgesehen. Schon kurz nach der NS-Machtübernahme wurden in Nürnberg etwa 250 Kommunisten verhaftet und ins rasch eingerichtete KZ Dachau verschleppt. Führende Sozialdemokraten erlitten das gleiche Schicksal. Die ersten Opfer in Dachau waren also deutsche Demokraten. Ab dem 30. August 1933 saßen nur noch Nationalsozialisten im Nümberger Stadtrat.

Auch wenn, wie gesagt, die NSDAP in Nürnberg in den Jahren vor 1933 keineswegs politisch dominierend war, so konnte sie nach der so genannten Machtergreifung auch in Nürnberg im Wesentlichen alles bestimmen. Daher kommt es, dass zwei Elemente des NS-Regimes - fortwirkend bis jetzt - mit dem Namen der Stadt eng verbunden sind, nämlich die Reichsparteitage der NSDAP und die sogenannten Nürnberger Gesetze.

\section{Die Reichsparteitage}

Die Entscheidung für Nürnberg als Austragungsort der ab 1933 alljährlich stattfindenden Reichsparteitage fiel im Juli 1933. Auch Stuttgart hatte sich darum beworben. Hitler favorisierte jedoch Nürnberg. Dahinter stand - neben dem Umstand, dass zur Durchführung einer solchen Massenveranstaltung die Infrastruktur einer Großstadt notwendig war - die Idee, an die Reichstage des Heiligen Römischen Reiches Deutscher Nation anzuknüpfen. In der sogenannten Goldenen Bulle des Jahres 1356 hatte Kaiser Karl IV. verfügt, dass jeder neu gewählte deutsche Kaiser, der in Frankfurt gewählt und in Aachen gekrönt werden sollte, in Nürnberg den ersten Reichstag abzuhalten habe. An diese alte Reichstradition, die allerdings im 16. Jahrhundert abbrach, und an das Renommee Nürnbergs, die „deutscheste der deutschen Städte“ zu sein, wollte Hitler anknüpfen. Vor dem historischen Hintergrund der Stadt wähnten sich die Nationalsozialisten als die Vollender der deutschen Geschichte.

Mit dem im Juli 1933 gegebenen Einverständnis des Nürnberger Oberbürgermeisters und kraft der Bestimmung durch Hitler, wonach Nürnberg der ständige Ort der Reichsparteitage ist, stand die Stadt im Dienst des NS-Regimes. Dessen Werbestrategen zogen eine direkte Linie von den Reichstagen des Mittelalters zu den jetzt - alljährlich im September stattfindenden - einwöchigen Staats- und Parteifeiern. Die NSDAP schuf sich mit dem Reichsparteitagsgelände eine riesige Versammlungsstätte. Rund eine Million Menschen nahmen jeweils als Aktive oder als bloße Zuschauer an diesem Spektakel, einer Mischung aus Machtdemonstration und Faszination, teil. Im Vordergrund der Veranstaltungen stand jeweils die Einschwörung der Massen auf den „Führer“ Adolf Hitler und die Inszenierung der propagierten ,Volksgemeinschaft".

Ein Architekt für das „Gigantenforum“ als „Weihestätte der Bewegung“ mit Aufmarschflächen, Versammlungs- und Lagerstätten fand sich in der Person des jungen Architekten Albert Speer, dem Adolf Hitler im Herbst 1934 den Auftrag erteilte, einen Gesamtplan für das Reichsparteitagsgelände zu entwerfen, das eine Größe von nicht weniger als 11 qkm umfasste.

Fertig gestellt wurden lediglich die Luitpoldarena, die 150.000 Teilnehmern aus dem Kreis der SS und der SA und zudem 50.000 Zuschauern Platz bot. Als weitere Anlage wurde das Zeppelinfeld vollendet. Es war eine fast quadratische Anlage mit Tribünen für rund 70.000 Zuschauer. Dieses Feld sein Name erinnert an die erste Landung des Luftschiffes „Zeppelin“ im Jahre 1909 - erlebte die Aufmärsche der politischen Leiter der NSDAP, des Reichsarbeitsdienstes, den „Tag der Gemeinschaft“ und Vorführungen der Wehrmacht.

Weitere Bauvorhaben blieben unvollendet: die Kongresshalle für 50.000 Teilnehmer, das Deutsche Stadion für 400.000 Besucher und das Märzfeld für Vorführungen der Wehrmacht, das bis zu 500.000 Besucher fassen sollte.

Der Architekt des Ganzen, Albert Speer, stand auch als Angeklagter vor dem Internationalen Militärtribunal in Nürnberg, freilich nicht in seiner Eigenschaft als Architekt des Führers, sondern als 
Rüstungsminister des Reiches (seit Februar 1942). Als solcher wurde er unter dem Aspekt des Völkerstrafrechts unter anderem zur Verantwortung gezogen wegen des Einsatzes von Zwangsarbeitern in der Rüstungsindustrie.

\section{Die sogenannten Nürnberger Gesetze}

Der Reichsparteitag des Jahres 1935 wurde zum Ort für die Verkündung von zwei Reichsgesetzen, die für alle deutschen Bürger jüdischer Abstammung in der Folgezeit katastrophale Folgen hatten. Diese Gesetze, das „Gesetz über das Reichsbürgerrecht“ und das „Gesetz zum Schutze des deutschen Blutes und der deutschen Ehre", waren zwar in den Berliner Ministerien erarbeitet, in Nürnberg jedoch abschließend beraten und verkündet worden. Deshalb werden sie umgangssprachlich heutzutage als Nürnberger Gesetze bezeichnet. Dieser Begriff ist nach wie vor ein Makel auf dem Namen der Stadt, obwohl die Kommune eigentlich eher beiläufig zum historisch-geographischen Ursprung dieser menschenverachtenden Normen wurde. Was war geschehen?

Ursprünglich hatte man keineswegs geplant, dieses Massenspektakel zum Fanal für die Degradierung jüdischer Mitbürger zu machen. Zwar war schon im Parteiprogramm der NSDAP (1920) die Forderung enthalten gewesen, dass nur ein Mensch ,deutschen Blutes“ auch deutscher Staatsbürger sein könne und jeder andere als Gast ,unter Fremdengesetzgebung" stehen müsse. Diese radikalen Forderungen der NSDAP bekamen aber erst nach der sogenannten Machtergreifung am 30. Januar 1933 „Oberwasser“. In der Folgezeit war man jedoch innerhalb der federführenden Ministerien - Innen- und Justizministerium - uneins, wie man diese Forderungen gesetzlich normieren solle. Diesem Tauziehen machte Hitler persönlich während des Reichsparteitages 1935 ein Ende. Es war geplant gewesen, am Abend des 15. September, des Abschlusstages, den formal noch bestehenden Reichstag, also das Parlament, einzuberufen und das vom Reichsinnenministerium vorbereitete Reichsflaggengesetz zu verabschieden, das die Hakenkreuzflagge zur Nationalflagge bestimmen sollte. Doch dies genügte Hitler für die als Höhepunkt des Reichsparteitages gedachte Reichstagssitzung nicht. Er gab daher am 13. September - also während des Parteitages, nur zwei Tage vor der geplanten Sitzung - dem Reichsinnenminister Frick den Auftrag, ,zur Auffüllung des Programmes“ ein Rassengesetz zu entwerfen, das der Reichstag neben dem Reichsflaggengesetz verabschieden sollte. Die Forderung nach einem Rassengesetz seitens der NSDAP war nicht neu - und Entwürfe gab es in den Schubladen der Berliner Ministerien schon etliche. Nun sollte binnen 24 Stunden alles perfekt sein. Die Staatssekretäre Pfundtner und Stuckart aus dem Reichsinnenministerium hatten die Vorlage für das Rassengesetz zu entwerfen. (Ein zeitlicher Vorgriff auf das spätere Schicksal der beiden: Pfundtner beging am 25. April 1945 in Berlin Selbstmord; Stuckart, der auch am 20. Januar 1942 an der Wannseekonferenz über die „Endlösung der Judenfrage" teilgenommen hatte, wurde durch ein US-Militärtribunal in Nürnberg am 11. April 1949 zu vier Jahren Haft verurteilt). Noch in der Nacht vom 13. zum 14. September wurde ein Mitarbeiter des Reichsinnenministeriums - Dr. Löser - von Berlin nach Nürnberg beordert. Die Genannten entwarfen dann anhand der vorhandenen Textvarianten die beiden Gesetze. Die erste Vorlage wurde von Hitler sofort abgelehnt und die Ausarbeitung von vier Entwürfen mit unterschiedlicher Schärfe befohlen. Es ging dabei nicht nur um die Staatsbürgerschaft, sondern auch um Berufs- und Gewerbebeschränkungen, um das Verbot der Heirat zwischen Ariern und Nichtariern, um Strafbestimmungen und anderes mehr. Die Ministerialbürokratie wollte den „mildesten“ Entwurf, der zudem nur "Volljuden“ betreffen sollte, bei Hitler „durchbringen“. Denn man befürchtete bei der Ausweitung des in Betracht kommenden Personenkreises Schwierigkeiten in der praktischen Umsetzung einer solchen Regelung. Letztlich entschied sich Hitler einerseits für die mildeste Form der Entwürfe. Er strich aber andererseits eigenhändig im Entwurf die Bestimmung, dass das Gesetz nur für „Volljuden“ gelten solle. Um dessen Außenwirkung - wohl auch mit dem Blick auf das Ausland etwas abzuschwächen, ordnete Hitler jedoch an, dass die Nachrichtenagentur DNB (,Deutsches Nachrichten-Büro“) melden solle, diese Regelungen würden nur für „Volljuden“ gelten.

Zwei Tage später, am Sonntag, dem 15. September, trat um $21 \mathrm{Uhr}$ im Jugendstilbau des „Kulturvereins“ der Reichstag unter dem Vorsitz des Präsidenten Göring zusammen. Neben dem Reichsflaggengesetz wurden sodann das sogenannte Reichsbürgergesetz und das Blutschutzgesetz 
beschlossen. Damit waren jüdische Bürger im NS-Regime erstmals rechtlich und s ozial zu Menschen zweiter Klasse geworden.

Wie scheinheilig man damals diesen Schritt zur Verfolgung deutscher Juden der deutschen Öffentlichkeit präsentierte, zeigt ein Bericht der für damalige Verhältnisse durchaus noch als "freie“ Presse zu wertenden „Bayerischen Volkszeitung“ vom 17. September 1935. Darin heißt es: „Bei einem Abschiedsabend im ,Deutschen Hof erklärte der Führer, dass den Juden in Deutschland nach diesen Gesetzen Möglichkeiten ihres völkischen Eigenlebens auf allen Gebieten eröffnet würden, wie sie bisher in keinem anderen Land zu verzeichnen wären. Im Hinblick darauf erneuerte der Führer den Befehl für die Partei, jede Einzelaktion gegen Juden wie bisher zu unterlassen". - In einem weiteren Bericht über diese gesetzliche Neuregelung steht zu lesen: „Indem Deutschland der jüdischen Minderheit Gelegenheit gibt, sich selbst zu leben und diesem Eigenleben (d.h. durch eigene Schulen, Theater, Sportverbände u.a.m.; d.Verf.) der jüdischen Minderheit den staatlichen Schutz gewährt, fördert es die Volkwerdung des Judentums und trägt dazu bei, das Verhältnis zwischen den beiden Nationen wieder erträglicher zu gestalten". Mit diesem Hinweis sollte ein Bezug hergestellt werden zu dem kurze Zeit vorher in Zürich abgehaltenen Zionistenkongress, der festgestellt hatte, dass das Judentum nicht nur eine Religion, sondern dass die Juden ein eigenes Volk seien.

Die Weltöffentlichkeit reagierte auf die Entrechtung jüdischer Bürger nicht sonderlich. Zwar gab es Aufrufe internationaler Sportverbände, deutscher Emigranten und anderer, die für das Jahr 1936 nach Deutschland vergebenen XI. Olympischen Spiele (Garmisch und Berlin) zu boykottieren. Das Internationale Olympische Komitee blieb aber bei seiner im Jahre 1931 getroffenen Entscheidung, obwohl sich die NSDAP unverblümt dagegen ausgesprochen hatte, Wettkämpfe gemeinsam mit „Negern und Juden“ durchzuführen. Wie wenig Resonanz die „Nürnberger Gesetze“ im politischen Leben der Völker fanden, erhellt geradezu beispielhaft die Tatsache, dass bei dem Reichsparteitag des Folgejahres - 1936 - mehr ausländische Diplomaten als früher in Nürnberg zu sehen waren. Und im Jahre 1937 nahmen erstmals auch die Botschafter Frankreichs und Großbritanniens, Francois-Poncet und Henderson, an der Selbstdarstellung des Nationalsozialismus in Nürnberg teil; die USA vertrat der Geschäftsträger ihrer diplomatischen Vertretung. Für hochrangige ausländische Besucher eröffnete der "Zweckverband Reichsparteitage“ im Jahre 1936 sogar ein eigenes „Diplomatenhotel“ unmittelbar neben dem renommierten Grand Hotel gegenüber dem Nürnberger Hauptbahnhof.

Nimmt man alles in allem, so war die Stadt Nürnberg ein Ort, an dem sich das nationalsozialistische System der Weltöffentlichkeit darstellte. Eine Legende ist es allerdings, dass eben deshalb die Alliierten im Jahre 1945 Nürnberg zum Sitz des Internationalen Militärtribunals gewählt hätten. Bei allen Vorgesprächen plädierten die Sowjets stets für Berlin, die ehemalige Reichshauptstadt, die sie erobert und besetzt hatten, auch wenn seit Juni 1945 den Westalliierten drei Sektoren der Stadt als Besatzungsgebiet zugeteilt waren. Die USA wollten den Prozess von Anfang an in ihrer Besatzungszone abhalten, denn sie organisierten und finanzierten alles. Auf der Suche nach einem geeigneten Gerichtsort ließ sich der spiritus rector des IMT, Robert H. Jackson, von dem stellvertretenden US-Militärgouverneur, General Lucius D. Clay, beraten. Jackson wusste, dass er ein weitläufiges Gebäude brauchte, da die Personalplanungen Raum für mehr als 1000 Mitarbeiter vorsahen. Auch sollte in nächster Nähe ein Gefängnis vorhanden sein, das nicht nur die Kriegsverbrecher selbst, sondern auch Tausende von Zeugen, die überwiegend aus Kriegsgefangenenoder Internierungslagern für längere Zeit nach Nürnberg gebracht werden mussten, aufzunehmen hatte. Clay stützte sich bei seinem Vorschlag an Jackson auf ein Gutachten des US-Army Generalanwalts Murray Barnays, der in Nürnberg den verhältnismäßig wenig kriegsbeschädigten Justizpalast und das nebenan gelegene Gefängnis besichtigt hatte. Nirgendwo anders in Deutschland fand sich eine solch ideale räumliche Konstellation. So einigte man sich schließlich mit den Sowjets in Artikel 22 des Statuts für den Internationalen Militärgerichtshof vom 8. August 1945 kompromissweise darauf, dass der ständige Sitz des IMT in Berlin ist, dass aber der erste Prozess, welcher wegen des Kalten Krieges der einzige blieb, in Nürnberg stattfindet.

Nürnberg trug viele Jahre schwer an der Bürde, dass der Name der Stadt weltweit mit dem Nationalsozialismus geradezu identifiziert wurde. Sieht man indes näher hin, kann man unschwer 
feststellen, dass Nürnberg nur e in e Station von etlichen auf dem mörderischen Weg eines Regimes war, das sich anschickte, Europa bis zum Ural unter seine Gewaltherrschaft zu zwingen.

\section{Literatur:} 2001).

Benz, Wolfgang, Hermann Graml und Hermann Weiß (Hrsg.), Enzyklopädie des Nationalsozialismus (München

Burleigh, Michael, Die Zeit des Nationalsozialismus - Eine Gesamtdarstellung (Frankfurt 2000).

Fritzsch, Robert, Nürnberg unterm Hakenkreuz - Im Dritten Reich 1933 - 1939 (Düsseldorf 1983).

Gruchmann, Lothar, „Blutschutzgesetz" und Justiz. Zu Entstehung und Auswirkung des Nürnberger Gesetzes vom 15. September 1935, in: VjZ 1983, 418.

Hambrecht, Rainer, Der Aufstieg der NSDAP in Mittel- und Oberfranken 1925 - 1933 (Nürnberg 1976).

Kulka, Otto Dov, Die Nürnberger Rassengesetze und die deutsche Bevölkenung im Licht geheimer NS-Lage- und Stimmungsberichte, in: VjZ 1984, 582.

Nadler, Fritz, Eine Stadt im Schatten Streichers (Nürnberg 1963).

Ogan, Bernd und Wolfgang W. Weiß (Hrsg.), Faszination und Gewalt. Zur politischen Ästhetik des Nationalsozialismus (Nümberg 1992).

Pohl, Dieter, Verfolgung und Massenmord in der NS-Zeit 1933 - 1945 (Darmstadt 2003).

Zelnhefer, Siegfried, Die Reichsparteitage der NSDAP in Nürnberg (Nürnberg 2002). 


\section{Klaus Kastner}

\section{In Retrospect: Nazi Party, the Rallies, the Racial Laws}

Decades after the National Socialist Germany had ceased to exist, Nuremberg continued to be remembered as a city, which had fulfilled a prominent role under the NS-regime. Although National Socialism in Nuremberg was not more predominant than elsewhere in Germany, the Nazi regime had skilfully misused Nuremberg's historical backdrop by turning it into a place that the Nazis used to mystically expose themselves as something empowered and revitalized.

The National Socialist Party (NSDAP) was founded 1920 not in Nuremberg, but Munich. However, Julius Streicher, an elementary school teacher and exceptionally repulsive anti-Semite, founded in 1922 a regional NSDAP group in Nuremberg. He "drummed up" enormous support for the Nazi movement. For over twenty years from 1923, he published in Nuremberg a series of nauseating anti-Semitic articles in "Der Stürmer" ("Storm Trooper"). The newspaper had a peak circulation close to 500,000 per issue. It thrilled its anti-Semitic readers with hair-raising stories about Jewish misconduct or crimes such as ritual child murder, rape, sinister political plots and so on. These anti-Semitic portrayals and demagogic campaigns would constitute an important step in mobilizing Germans to exterminate a whole group of people because they were Jews. Streicher was found guilty by the IMT and sentenced to death by hanging.

In summer of 1933, Hitler chose Nuremberg to be the permanent site for the NSDAP Annual Party Rally. He preferred this large town equipped with a more than adequate infrastructure to accommodate millions of spectators because he believed Nuremberg was the "most German of all German cities" by taking into consideration the predominant position Nuremberg had within the Holy Roman Empire of the German Nation and using it for his propaganda.

The early political claims of the NSDAP were transformed into law with the passage of the socalled Nürnberger Rassengesetze (Nuremberg Racial Laws). "Aryan" descent should become the prerequisite for citizenship in the German Reich. Hitler personally ordered at short notice that the laws should be proclaimed during the 1935 Party Rally in Nuremberg. Within two days, drafts of the "Citizens' Rights Act" and the "Protection of German Blood and German Honour Act" were prepared and subsequently passed by a special session of the Reichstag (parliament) in Nuremberg on September 15, 1935. Regardless of the legal discrimination against Jews, the XI Olympic Games were anyways held in Berlin the following year. At subsequent party rallies the number of diplomats and guests from abroad attending the procession were even higher than the years previously!

It was a heavy burden for Nuremberg that worldwide the name of the city was seen as a synonym for National Socialism. Nuremberg was only one of many stations along the murderous path of a totalitarian regime preparing to tyrannically and coercively control Europe to the Ural. 